La revue La revue pour l'histoire du CNRS

POUR LHISTOIRE DU CNRS $\quad 17 \mid 2007$

Objectif biotechs?

\title{
Des biotechnologies en bonne santé
}

Pierre Tambourin

\section{(2) OpenEdition}

Journals

Édition électronique

URL : https://journals.openedition.org/histoire-cnrs/1913

DOI : 10.4000/histoire-cnrs.1913

ISSN : 1955-2408

Éditeur

CNRS Éditions

Édition imprimée

Date de publication : 3 juillet 2007

ISBN : 978-2-271-06558-2

ISSN : 1298-9800

\section{Référence électronique}

Pierre Tambourin, «Des biotechnologies en bonne santé », La revue pour l'histoire du CNRS [En ligne],

17 | 2007, mis en ligne le 03 juillet 2009, consulté le 20 mai 2021. URL : http://

journals.openedition.org/histoire-cnrs/1913 ; DOI : https://doi.org/10.4000/histoire-cnrs.1913

Ce document a été généré automatiquement le 20 mai 2021.

Comité pour l'histoire du CNRS 


\title{
Des biotechnologies en bonne santé
}

\author{
Pierre Tambourin
}

1 Le terme " génie génétique » est né dans les années 1970, peu après la découverte des enzymes de restriction, ces ciseaux moléculaires capables de reconnaître des motifs plus ou moins longs, très spécifiques sur la molécule d'ADN, puis de couper de manière très précise cette même molécule. D'autres outils moléculaires, comme les ligases, les polymérases constituent très rapidement une véritable caisse à outils pour le biologiste moléculaire et donnent à la biologie deux impulsions décisives :

- Une science essentiellement descriptive devient, en quelques années, beaucoup plus explicative.

- Une science d'intérêt industriel modeste, bien que déjà significatif, particulièrement en agriculture, révèle un potentiel exceptionnel et surtout imprévu. L'ère des biotechnologies industrielles modernes démarre avec la création de la société Genentech (San Francisco) en 1978, la production de l'hormone somatostatine et de l'insuline humaine en 1978 (mise sur le marché en 1982). Ces avancées rapprochent le monde des financiers de celui des laboratoires de recherche académique ce qui, s'agissant du vivant, allait très vite poser des problèmes sociétaux difficiles et nombreux dont les OGM constituent aujourd'hui l'archétype le plus visible.

Dès 1975, anticipant ces questions, les chercheurs du monde entier les plus en pointe de l'époque s'interrogent, lors du fameux congrès d'Asilomar, sur leurs responsabilités et les enjeux sociétaux de ces recherches. Un peu naïvement fut décidé un moratoire logiquement peu respecté. En effet, comment répondre à ces interrogations si tout travail de recherche permettant d'y répondre est arrêté ? Le moratoire est d'ailleurs levé dès 1976. Des règles assez strictes sont rédigées.

Le contexte historique

3 Au plan international, l'émergence des biotechnologies modernes remonte à l'ère du président Nixon qui, à l'image de John F. Kennedy et de la conquête de la lune, veut marquer sa présidence par le lancement d'un programme très ambitieux. Ce fut le Plan Cancer qui se proposait d'éradiquer cette terrible maladie en cinq ans par l'apport de moyens financiers publics (et privés) sans précédent en biologie. Le cancer ne fut pas vaincu, mais ces budgets, faramineux pour l'époque, propulsent la biologie américaine 
à un niveau de performance et d'excellence qu'elle n'a d'ailleurs pas quitté depuis. Les États-Unis consacrent toujours aux recherches biomédicales au sens large près de trois fois plus de budgets par habitant que la plupart des autres puissances mondiales.

Que se passe-t-il pendant ce temps en France?

4 En 1958, le Général de Gaulle arrive au pouvoir. Il tient le discours que l'on connaît sur la grandeur et le rôle de la France dans le monde. Il comprend très vite le rôle et l'importance de la recherche. Il crée le Comité des Sages puis la DGRST (Délégation générale à la recherche scientifique et technique) et lance, entre autres, un programme ambitieux en biologie moléculaire. Celui-ci permit aux dispositifs français de recherche en biologie de se moderniser rapidement. En effet, à cette époque, en dehors de quelques rares laboratoires dont ceux de François Jacob, Jacques Monod et André Lwoff de l'Institut Pasteur, la biologie française paraît bien poussiéreuse. Les disciplines classiques, purement descriptives, pèsent sur le système et par exemple, l'approche génétique des phénomènes biologiques a bien du mal à percer alors qu'elle est déjà performante dans d'autres pays.

5 Soucieux de rester indépendant dans des domaines fortement stratégiques, Charles de Gaulle, appuyé par les grands corps de l'État, lance avec des moyens financiers publics importants les grands programmes mobilisateurs qui façonnent notre industrie et structurent notre recherche. Sont ainsi lancés ou renforcés les programmes du nucléaire civil et militaire : "Concorde » suivi des grands programmes de transports (Airbus, TGV), le spatial, l'informatique, les télécommunications. Tout ne fut pas couronné de succès - le Plan Calcul par exemple -, mais nombre de ces programmes permirent à la France d'après-guerre de retrouver une place enviable dans des secteurs industriels de haute technologie. Ces choix politiques restent encore aujourd'hui très présents. Ils expliquent en partie l'incapacité de notre pays à comprendre les enjeux de même nature véhiculés par les sciences de la vie. Pire encore, l'importance industrielle de très jeunes entreprises innovantes, que ce soit en biotechnologie ou dans d'autres disciplines, fut ignorée. La création récente (2005) de l'Agence de l'innovation industrielle en contrepoids de l'ANR est, à cet égard, très instructive.

6 Cette période, très féconde, aurait probablement permis à la France de se positionner en sciences du vivant si un coup d'arrêt brutal n'avait été donné à cette politique ambitieuse de recherche après le départ du Général de Gaulle. Seuls les progrès industriels d'intérêt stratégique furent maintenus, voire accentués, dans la décennie suivante avec les conséquences désastreuses sur l'attractivité de ces métiers pour les jeunes et l'exode massif des post-doctorants vers des carrières différentes ou plus simplement vers les États-Unis.

7 Ce pays va poursuivre ses efforts pendant les décennies suivantes et attirer nombre de jeunes chercheurs étrangers bien formés dans leurs pays, prêts à renforcer les laboratoires, en particulier dans les sciences du vivant. La France, de son côté, essaie de tirer profit de ses singularités ; Jacques Monod en particulier, attire dans son laboratoire normaliens, polytechniciens et centraliens. Après une première vague constituée de Philippe Lazar, Joseph Lellouche, qui autour de Daniel Schwartz, le frère du mathématicien Laurent Schwartz, apporte à la médecine une assise méthodologique plus solide par l'usage des statistiques, une seconde vague entre de plein pied dans la biologie moléculaire.

8 Citons par exemple Maxime Schwartz, Philippe Kourilsky, mais il y en eut beaucoup d'autres. Ceci ne produit d'ailleurs pas de grands changements au sein de ces grandes 
écoles où aujourd'hui encore la biologie est soit absente, soit très marginalisée. Seules les Écoles normales supérieures (Ulm, Cachan, Lyon) et l'École centrale s'ouvrent sérieusement à la biologie au cours de cette longue période ${ }^{1}$.

Le CNRS générateur des connaissances?

9 À côté de l'Institut Pasteur, le CNRS, ou plutôt les scientifiques du CNRS, jouèrent un rôle clé dans l'émergence de la biologie moléculaire en France, sans prendre le virage emprunté par les États-Unis ou l'Angleterre qui valorisent la recherche par la création de jeunes entreprises innovantes en biotechnologie. Pire, le CNRS ne comprend pas l'importance cruciale qu'allait prendre dans ces domaines le brevet, ou plus généralement, la protection intellectuelle et industrielle. Quelques chercheurs essayent de s'y lancer comme Pierre Potier (Navelbine ${ }^{\circ}$, Taxotère), Luc Montagnier (HIV), Francis Galibert (hépatite) mais la vaste majorité des chercheurs, soit par principe, soit par ignorance, soit en raison des lourdeurs administratives, continuèrent et continuent à privilégier les publications scientifiques de très bon niveau.

Or, sans protection intellectuelle ni brevet, il n'y a pas de possibilité de valorisation de la recherche. Dans les domaines où de grands industriels participent aux travaux des laboratoires, le portefeuille de brevets leur est confié. Dans les sciences du vivant, les industriels n'existent pas - l'industrie pharmaceutique est à l'époque une activité essentiellement chimique -, c'est aux organismes publics de le faire. Ce que l'Inserm, l'Inra ou le CEA ont d'ailleurs en partie compris et beaucoup mieux fait. L'Inserm a créé très vite un véritable service de valorisation qui a produit récemment InsermTransfert, structure professionnalisée au service des laboratoires, des chercheurs, de l'organisme (ce que font les universités américaines depuis fort longtemps).

Les prises de conscience ... fugitives

11 En 1983-84, l'Inserm entre dans le capital de quelques sociétés anonymes², Pasteur crée Transgène, la direction générale du CNRS semble enfin prendre en compte l'importance des biotechnologies et Roger Monier et Claude Fréjacques proposent, par exemple, la création d'une « joint-venture » (filiale) en agro alimentaire. Mais les compétences font défaut et le choix n'est guère pertinent pour le CNRS, les autres départements freinent cette action et cette volonté qui durent peu de temps.

12 En 1993, Guy Aubert réfléchit avec moi à nouveau à cette question et, en dépit des excellentes relations entre nous et le poids des arguments du directeur du département, Guy Aubert refuse de se lancer dans cette aventure pour des raisons conjoncturelles qui, aujourd'hui, paraissent recevables : pas de service de valorisation interne digne de ce nom (la société FIST est une filiale de plusieurs organismes). De plus, la France traverse une période économique très difficile, le statut des chercheurs s'oppose à la prise de participation au capital et l'argent spécifiquement consacré à ses entreprises manque.

13 Par ailleurs, l'ambiance générale n'est pas encore ce qu'elle allait devenir cinq années plus tard. Enfin, dans la vision défendue énergiquement par les partenaires sociaux du CNRS qui ne peuvent imaginer un CNRS autrement que comme un bloc uni et indivisible, voir un département se lancer dans une telle politique paraissait une véritable provocation. Au total, au cours de cette longue période de près de vingt années, deux directeurs scientifiques du département des sciences du vivant auront essayé de faire évoluer le CNRS en nommant des chargés de mission en biotechnologie : Roger Monier avec Maurice Hoffnung (autre polytechnicien) et moi-même avec Bernard Pau qui avait créé une société avant de devenir lui-même directeur du 
département. Aucune autre initiative de ce type ne fut prise dans aucun département, aucun service de brevet digne de ce nom ne fut créé, aucune structure de valorisation à la hauteur des enjeux ne fut même imaginée. FIST a toujours été et reste encore de taille insuffisante.

L'emprise des syndicats

L'histoire des relations recherche publique/industrie privée a toujours été marquée par une approche fortement idéologique. Avant 1980, - cette date n'est probablement pas une coïncidence -, chercheurs, ingénieurs, techniciens et leurs syndicats restent, en très grande majorité, hostiles à tout rapprochement avec l'industrie. À cela, plusieurs raisons très vivement ressenties par les acteurs du CNRS :

- Un dialogue même transitoire avec un industriel - l'aval comme il est dit -, ne peut que pervertir la démarche logique intrinsèque de la recherche fondamentale, donc ne peut que la retarder et par conséquent, l'handicaper. Il suffit de regarder l'histoire des sciences et la pratique d'autres pays pour démontrer simplement que si la recherche dite fondamentale ne peut pas être seulement conditionnée par la demande aval, les liens qui se créent entre les divers partenaires naturels des chercheurs (industriels, médecins, associations de malades, et plus généralement la société), peuvent être riches de développements novateurs, y compris dans la recherche la plus fondamentale.

- Le rôle du chercheur du secteur public est d'être évidemment au service de l'intérêt général, du plus grand nombre. De plus, pour cette raison d'ailleurs, la science est essentiellement un tissu d'individus qui collaborent au plan international. Par conséquent, travailler avec un partenaire privé ne peut qu'aliéner cette mission de service public. Le chercheur du secteur public ne peut participer du fait de cette mission et de son statut à cette marchandisation du savoir de plus en plus pressante, voire violente. Sauf qu'une telle vision n'a aucun sens par rapport à l'essence même de la mission de la recherche publique qui est de participer au développement économique du pays qui lui permet de se développer et cela ne peut passer évidemment que par les entreprises nationalisées. La bonne réponse à cette difficile question est, bien entendu, de veiller soigneusement au respect des droits et des devoirs de chacun dans ces nécessaires collaborations. C'est par manque de maîtrise de ces systèmes de contrôle que, bien souvent, les chercheurs se réfugient dans ce qu'il est convenu d'appeler leur tour d'ivoire. Après 1981, les relations s'améliorent mais l'industriel reste pour l'essentiel un partenaire qui permet aux laboratoires publics de disposer de financements complémentaires. L'intérêt heuristique ou stratégique de ces relations est largement ignoré du monde de la recherche, il faudra attendre la période 2000-2005 pour noter un changement important de paradigme dans ce domaine.

Le statut des organismes Jean-François Théry, membre du Conseil d'État, a participé avec d'autres à l'élaboration des textes qui fondèrent la loi d'Orientation de 1982. Dans un ouvrage récent ${ }^{3}$, écrit avec Rémi Barré, il raconte comment et sur quelle base idéologique nouvelle, cette loi fut conçue. Il indique comment les grands syndicats de fonctionnaires, en particulier la CGT des PTT, eurent en permanence le souci de réduire à néant les efforts faits pour, d'une part, tenir l'engagement de " fonctionnariser » les chercheurs, et d'autre part, de ne pas tomber dans le travers des statuts de l'administration publique. Il s'agissait de créer un statut dérogatoire, en partie seulement obtenu, et qui explique d'ailleurs, aujourd'hui encore, la lourdeur de la gestion dans ces organismes. Ce texte fut très novateur mais il est resté très éloigné de ce que souhaitaient les acteurs de l'époque, y compris les représentants des syndicats de chercheurs. Cette loi essayait en particulier 
de lancer les bases d'un véritable partenariat public/privé en affirmant le rôle des organismes de recherche dans la valorisation. Ceci eût été possible si, d'une part, la culture du milieu avait permis de développer une véritable action dans ce domaine et si, d'autre part, le statut des organismes avait facilité la création de structures filiales adaptées à ces missions. Cependant, cette avancée, même très partielle, peut expliquer l'engouement de la recherche publique accompagnée par certains industriels autour des biotechnologies. Celles-ci explosent littéralement aux États-Unis à l'époque, comme le montre la création, en 1982, en Californie, de la société AMGEN, aujourd'hui première entreprise de biotechnologie mondiale, $3 e$ dans le monde de l'industrie pharmaceutique. L'Institut Pasteur, l'Inra, l'Inserm s'impliquèrent dans quelques créations mais en nombre dérisoire. Rien de tel au CNRS, toujours pour des raisons idéologiques. Brusquement, par ces effets de mode dont notre pays (et d'autres) est coutumier, l'effort s'arrête brutalement. Il faut dire que chez les grands industriels de l'industrie pharmaceutique, la mode était devenue le «me-too» et la stratégie de l'époque affirmait que la compétence forte en chimie interdisait d'investir en biologie.

16 Et comme il faudra bien, à un moment ou à un autre, revenir à la chimie, il suffit d'attendre tranquillement que les États-Unis investissent en biologie et nous permettent de revenir au moment du retour indispensable vers la chimie. L'idée est de laisser aux États-Unis les efforts en ce domaine et il suffit d'observer ce qui s'est passé dans l'industrie pour se rendre compte de l'erreur d'appréciation. Au ministère de l'Industrie, personne ne s'occupait véritablement de ces questions. Seul le ministère de la Recherche, avec des personnalités comme Daniel Thomas, professeur à l'université de Compiègne, Gilbert Durand, professeur à l'Insa, et Pierre Douzou, directeur de recherche à l'Inserm, a essayé de mobiliser les forces mais avec bien peu de succès. Bien entendu, la machine mise en marche en 1978 aux États-Unis poursuit sa progression fulgurante.

En Angleterre, l'ère Thatcher, très difficile pour la recherche publique, se caractérise par une mobilisation autour des biotechnologies. Puis l'Allemagne près de dix ans plus tard, suivant le mouvement d'ensemble de toute l'Europe du nord, investit dans ce domaine. La France est toujours hésitante entre la position des pays du nord et ceux du sud. C'est seulement entre 1995 et 2000 que les gouvernements successifs donnèrent une impulsion décisive à ce domaine.

Comment comprendre cette politique française?

18 L'une des premières causes probables de l'« autisme » français dans ce domaine, alors qu'une mobilisation de grande ampleur se met en place dans les pays voisins et dans le monde entier, tient probablement à la division historique entre les grandes écoles et les universités. Les grandes écoles sélectionnent une élite qui n'a souvent aucune formation par la recherche. Or, l'innovation naît de la recherche et implique une forte connaissance de ce monde. Aux États-Unis par exemple, près de $50 \%$ des cadres dirigeants d'entreprises ont obtenu un $\mathrm{PhD}$, ce qui n'est pas le cas en France. En particulier, dans les grands corps de l'État dont on connaît l'influence dans la politique industrielle de notre pays, l'absence de culture scientifique en sciences du vivant est inquiétante.

19 La sélection s'appuie le plus souvent sur des disciplines dites dures, ce qui se traduit évidemment par une faible connaissance des enjeux dans les autres disciplines. L'industrie pharmaceutique est d'ailleurs vue également plus comme du commerce (business) que comme un enjeu industriel ou culturel (politique de santé). Les médecins 
quant à eux, qui forment une autre élite, sont restés longtemps ignorants de cette partie très dure de la biologie, à l'exception, bien entendu, des médecins chercheurs de l'Inserm ou de ceux qui ont eu la chance de soutenir une thèse en sciences.

Enfin, les grands industriels directement concernés par ces développements étaient chimistes de formation ou pharmaciens et restaient eux aussi éloignés de la biologie moléculaire. Lors du grand programme " Essor des biotechnologies », le CNRS brille surtout par son absence et affiche clairement dans ses priorités stratégiques les sciences pour l'ingénieur. En budget de fonctionnement consolidé (hors salaires) incluant de très grands équipements ou autres actions mutualisées, un chercheur de l'IN2P3 recevait, il y a 10 ans, 10 fois plus de moyens qu'un chercheur des sciences du vivant et plus de 15 fois qu'un chercheur des sciences de l'homme ou de la société. Il ne s'agit pas, bien entendu, d'élever les disciplines les unes contre les autres mais simplement de traduire par des chiffres des choix souvent justifiés par l'absence de moyens de fonctionnement suffisants : les chercheurs en physique n'ont évidemment pas d'association caritative pouvant les aider. Devant les questions récurrentes des chercheurs des départements des sciences du vivant, la direction de la stratégie de l'époque répond sans humour que les choix politiques aux États-Unis sont liés aux activités de lobbying bien connues du NIH américain, et qu'heureusement, la France et le CNRS échappent à de telles pressions " malfaisantes " pour la science et pour le développement normal de notre pays. D'ailleurs, les sénateurs ne sont-ils pas aux ÉtatsUnis hypersensibles aux problèmes de santé vu leurs âges !!!

L'avenir

21 Aujourd'hui, les insuffisances du passé sont en cours de correction ou ont été en partie corrigées soit en interne, soit à l'extérieur du CNRS. Après l'audit de la Cour des comptes, le CNRS a changé d'attitude en matière de brevet et si, aux dires des chercheurs et en particulier dans les sciences du vivant, il y a encore beaucoup à améliorer, le choix politique semble définitif. Preuve en est donnée par le nombre croissant d'entreprises en haute technologie que le CNRS et/ou l'université engendrent en s'appuyant sur des incubateurs publics ou sur Genopole à Évry. Genopole regroupe aujourd'hui un portefeuille de 62 entreprises dont 9 sont issues du CNRS.

Un peu plus de professionnalisme, un peu plus d'exigence, un peu plus de moyens aussi consacrés à ces activités et le CNRS sera définitivement plus performant dans ces domaines. D'ailleurs, le CNRS fait face aujourd'hui à un autre défi de grande importance au moins égal, sinon plus, à celui qui vient d'être longuement évoqué et qui touche lui aussi aux sciences du vivant.

23 Ce domaine a récemment développé des technologies nouvelles dites de la biologie à grande échelle qui permettent de suivre par exemple l'expression des 25000 gènes présents dans le génome de l'homme dans des cellules, des organes au cours du temps et de comparer la situation entre état normal et état pathologique. La plupart de ces technologies exigent pour leur développement des approches très sophistiquées dont les compétences se retrouvent dans les nanotechnologies, la micro fluidique, l'optique, l'informatique. Par ailleurs, stocker, gérer, exploiter des quantités de données qui doublent tous les sept mois pose de véritables problèmes.

Enfin, l'approche par la modélisation, la simulation, les concepts issus d'autres sciences, des problèmes qui concernent la santé de l'homme, la biodiversité des espèces, le fonctionnement du cerveau, l'équilibre des écosystèmes deviennent une exigence dont chacun comprendra qu'elle ne peut se réduire à un discours velléitaire. Le CNRS est 
l'endroit par excellence où ces approches doivent se mettre en place, en liaison avec le CEA et les universités, en appui aux organismes plus finalisés, plus mono disciplinaires que sont par exemple l'Inserm et l'Inra. Un enjeu considérable qui touche aux missions de services publics en recherche fondamentale du CNRS et que ces chercheurs, de qualité, sont capables d'assumer, à condition que cette grande maison veuille bien se mobiliser, et surtout, qu'on lui donne les moyens d'atteindre cet objectif.

\section{NOTES}

1. Sans oublier l'Insa de Toulouse (UMR 5504) qui, sous l'impulsion du professeur Gilbert Durand, a joué un rôle déterminant dans le développement des technologies liées aux bioprocédés.

2.Notamment la société Immunotech, émanation du CNRS et de l'Inserm sur le campus de Marseille-Luminy.

3. La loi sur la recherche de 1982. Origines, bilan et perspectives du "modèle français ». JeanFrançois Théry, Rémi Barré, Éditions Inra, collection Sciences en questions, 2001.

\section{RÉSUMÉS}

Les trente dernières années ont connu trois révolutions majeures dans le domaine de la biologie française : l'apparition de la génétique moléculaire ; la création d'entreprises innovantes proches de l'industrie pharmaceutique ; le lancement des programmes de séquençage du génome humain. Pierre Tambourin dresse un bilan de cette « spirale » intellectuelle et scientifique.

Since thirty years, biology had known three major revolutions: the birth of molecular genetics; the creation of pharmaceutical companies; the launch of human genome sequence programs. Pierre Tambourin has lived these periods as a real pioneer. Here is his report.

\section{AUTEUR}

\section{PIERRE TAMBOURIN}

Ancien élève de l'École polytechnique, spécialiste en cancérologie moléculaire, chercheur à l'Inserm, Pierre Tambourin est, depuis 1998, directeur général de Genopole à Évry. Il préside en outre, depuis 1998, le Conseil scientifique de l'Institut national de la recherche agronomique (Inra). De 1993 à 1997, il a dirigé le département des sciences de la vie du CNRS et, de 1989 à 1992, la section Recherche de l'Institut Curie. 